\title{
Dynamical Renormalization of Kinetic van der Waals Spin Models
}

\author{
Attilio Stella ${ }^{1,2}$ and Raf Dekeyser ${ }^{1}$
}

Received July 11, 1980

\begin{abstract}
An exact dynamical renormalization approach in differential form is proposed for kinetic van der Waals spin systems with general many-body interactions. The problem of restoring covariance in the evolution equation after renormalization of the model is solved by introducing a suitable renormalized time parameter, which depends also on the magnetization of the spin configuration. The study of the behavior of this renormalized time near criticality leads to a scaling relation for the linear relaxation time. This relation can be shown to imply the exact results for the dynamical critical behavior of the system.
\end{abstract}

KEY WORDS: van der Waals spin systems; molecular field; critical dynamics; differential renormalization.

\section{INTRODUCTION}

When trying to extend to the dynamical context the static renormalization approach to critical phenomena, the relevant new problem arises of mapping the given time evolution law in a covariant way into a new law, appropriate for the coarse-grained system. Such a problem is in general highly nontrivial; this is the main reason why there has been relatively little progress in the direction of applying so-called real-space renormalization techniques ${ }^{(1)}$ to lattice spin systems with dynamics governed by a master equation. ${ }^{(2)}$ The action of block-spin transformations on these systems, for example, unavoidably introduces memory effects which, if not handled

\footnotetext{
${ }^{1}$ Instituut voor Theoretische Fysica, Katholieke Universiteit Leuven, Celestijnenlaan 200 D, B-3030 Leuven, Belgium.

${ }^{2}$ On leave of absence from Instituto di Fisica e Unità G.N.S.M. del C.N.R., Università di Padova, Padova, Italy.
} 
systematically, destroy the covariance under renormalization of the time evolution equation, thus depriving in principle the whole method of its effectiveness.

Especially in view of our still poor ability in facing the general difficulties connected with the implementation of a dynamical renormalization program, it seems to us of considerable theoretical interest to study model systems, which are simple enough to allow for a complete and satisfactory realization of such a program without approximations.

In the present article we propose and elaborate exactly a renormalization scheme for mean field, van der Waals systems with stochastic dynamics. As far as the statics is concerned, this renormalization method was discussed in a previous publication by the present authors. ${ }^{(3)}{ }^{3}$ The scheme has the peculiar technical feature of being of a differential type, which proved to be extremely powerful and elegant in extracting all the thermodynamic and critical properties of the model. While the van der Waals systems are relatively trivial in themselves and have some unrealistic physical features, the static differential renormalization procedure applied to them proved to be very interesting and allowed the explicit calculation of some quantities, which could not be otherwise obtained by more standard renormalization approaches. Most important, in our opinion, those calculations provided an exact prototypical realization of some of the features that an analogous approach should show when applied to more complicated systems.

A strong motivation in that work was also given to us by the hope that the efficiency of the differential technique could allow for a relatively easy dynamical extension of the approach, to which the present article is devoted.

Using our differential scheme, we are able to follow explicitly how the dynamics of the model is modified as a consequence of an infinitesimal decimation of the degrees of freedom. We then succeed in taking into account this modification, together with the accompanying covariancebreaking effects, in a very simple and elegant way, by a suitable redefinition of the time parameter. This completely restores the original form of the evolution equation. The knowledge of the transformation of the time under renormalization leads finally to the possibility of predicting the behavior of the characteristic relaxation time of the system as a function of temperature and magnetic field, using only scaling arguments. Consistently with the general attitude inspiring the renormalization approach, the results are not obtained by solving directly the time evolution of the system, but only by

\footnotetext{
${ }^{3}$ Hereafter we refer to this paper as $I$.
} 
exploiting properly the properties of the mapping of this evolution into the renormalized evolution.

The paper is organized in five sections, the second of which is devoted to the introduction of the model and the discussion of the main features of its dynamics. Some of the basic concepts and results of I are briefly summarized in Section 3, where our dynamical renormalization program is clearly stated. There we also solve explicitly the problem of restoring covariance in the equation of motion. The renormalized time function, introduced for this covariance, is further discussed in Section 4, and the dynamical scaling indices are obtained on the basis of the fixed point behavior. Section 5 contains some concluding remarks. An Appendix is devoted to a detailed study of the renormalized time function and of the scaling corrections. This fully justifies some assumptions made for the derivation in Section 4.

\section{THE MODEL AND ITS DYNAMICS}

As in Paper I, we are dealing here with classical spin systems having arbitrary many-body, mean field interactions. With the same notations, we will write the reduced Hamiltonian $\beta H$ as a function of the average magnetization $m$ of the given configuration $\{S\}$ of the set of $N$ spins:

$$
-\beta H(\{S\})=N e(m)
$$

where $\beta=1 / k_{B} T$ and

$$
m=N^{-1} \sum_{j=1}^{N} S_{j} \quad\left(S_{i}= \pm 1\right)
$$

In the limit when $N$ goes to infinity, $m$ becomes a continuous variable.

The equilibrium canonical probability distribution for the configurations can be written as

$$
P_{0}(\{S\})=Z^{-1} e^{-\beta H(\{S\})}=Z^{-1} e^{N e(m)}=\bar{P}_{0}(m)
$$

with the partition function

$$
Z=\operatorname{Tr}_{\{S\}} e^{N e(m)}
$$

$\operatorname{Tr}_{\{s\}}$ indicates a trace over all $2^{N}$ configurations. In the following $e(m)$ is assumed to be a general, sufficiently regular function, defined on the interval $(-1,1)$.

A kinetic version of a mean field spin model was first studied by Griffiths et al. ${ }^{(4)}$ in connection with the problem of the relaxation time of metastable states. Here we introduce the time evolution in a completely analogous way, by using a master equation with single spin-flip transitions. 
We denote by $P(\{S\}, t)$ the time-dependent nonequilibrium probability of the configuration $\{S\}$, and we assume the evolution equation

$$
\begin{aligned}
\frac{\partial}{\partial t} P(\{S\}, t)= & -\sum_{i=1}^{N}\left\{W_{i}\left(\left\{(S)_{i}, S_{i}\right\}\right) P\left(\left\{(S)_{i}, S_{i}\right\}, t\right)\right. \\
& \left.-W_{i}\left(\left\{(S)_{i},-S_{i}\right\}\right) P\left(\left\{(S)_{i},-S_{i}\right\}, t\right)\right\}
\end{aligned}
$$

By $(S)_{i}$ we have indicated the set of all spins except the $i$ th one, and thus $\left\{(S)_{j},-S_{j}\right\}$ represents the configuration obtained from $\{S\}=\left\{(S)_{j}, S_{j}\right\}$ by reversing the $j$ th spin. $W_{j}(\{S\})$ describes the probability per unit time for the flipping of the $j$ th spin from $S_{j}$ into $-S_{j}$, given a certain configuration $\{S\}$.

The choice for $W_{j}$ is of course not unique; here we choose

$$
W_{j}(\{S\})=P_{0}(\{S\})^{-1 / 2} P_{0}\left(\left\{(S)_{j},-S_{j}\right\}\right)^{1 / 2}
$$

which satisfies the detailed balance condition, thus guaranteeing the ergodicity of the system.

Like the Hamiltonian, also the nonequilibrium probability distribution of the configurations will be assumed to depend only on the mean magnetization $m$, and it will be written as

$$
P(\{S\}, t)=\bar{P}(m, t)=Z^{-1} \exp \{N(e(m)+h(m, t))\}
$$

$h(m, t)$ thus measuring the time-dependent deviation from the equilibrium distribution. Since the $Z$ appearing in (7) is the time-independent partition function, the function $h(m, t)$ must embody an $m$-independent term such that

$$
\operatorname{Tr}_{\{S\}} P(\{S\}, t)=1
$$

The way in which our system approaches equilibrium will be fully described by the time dependence of $h(m, t)$.

The time evolution of $h$ is easily obtained in the thermodynamic limit, when we let $N$ go to infinity. The discrete variable $m$ becomes continuous in this limit, and (5) can easily be translated into a partial differential equation for $h$. Indeed, starting from (5) and taking into account that the magnetization of a given configuration $\{S\}$ changes from $m$ to $m-$ $\left(2 S_{j} / N\right)$ after flipping the $j$ th spin, one obtains with simple algebra, for $N$ going to infinity,

$$
\frac{\partial h}{\partial t}=-\cosh \dot{e}+m \sinh \dot{e}+\cosh (\dot{e}+2 \dot{h})-m \sinh (\dot{e}+2 \dot{h})
$$

where the dots indicate partial derivatives with respect to $m$. After differen- 
tiation with respect to $m$ on both sides, (9) becomes a quasilinear partial differential equation for $\dot{h}(m, t)$.

In what follows, we will work with the linearized version of (9), in which only first-order contributions in $h$ are taken into account. This is sufficient as far as the discussion of the linear relaxation of the system is concerned. This linearized version of (9) can be written in the form

$$
\frac{\partial h}{\partial t}=A(\dot{e}, m) \dot{h}
$$

with

$$
A(f, m)=2(\sinh f-m \cosh f)
$$

The specific form of $A$ is of course a consequence of the choice we made in (6) for the transition probabilities. In view of further considerations, it is interesting to notice that an equally acceptable choice would be to multiply the right-hand side of Eq. (6) by an arbitrary positive nonsingular function of $(S)_{j}$. If we are interested in an equation still leading to an evolution completely describable in terms of the variables $t$ and $m$ alone, then the dependence on $(S)_{j}$ can be assumed through the argument $\left(m-S_{j} / N\right)$, which is $S_{j}$ independent. One can easily check that if we call $a\left(m-S_{j} / N\right)$ the above function, then the coefficient $A$ in the final equation (10) is replaced by

$$
A^{\prime}(\dot{e}, m)=a(m) A(\dot{e}, m)
$$

Equation (10) can be seen to have the general solution

$$
h(m, t)=h_{0}\left(\tilde{m}_{0}(m, t)\right)
$$

$\tilde{m}_{0}(m, t)$ is the inverse of $\tilde{m}\left(m_{0}, t\right)$, which is itself the solution of the characteristic equation

$$
\frac{d m}{d t}=-A(\dot{e}(m), m)
$$

with the initial value $m(t=0)=m_{0}$. The function $h_{0}$ is given by the initial condition on the deviation from equilibrium:

$$
h_{0}(m)=h(m, 0)
$$

From the solution (13) all the dynamic information on the infinite system can be obtained in first order in the deviations from equilibrium. The evolution of the average magnetization, in particular, can be obtained straightforwardly by a standard saddle point argument for this $N$ infinity situation. We mention that a more extended and formally rigorous discussion of models within the class discussed here can be found in Ref. 5. 


\section{INFINITESIMAL TRANSFORMATIONS AND THEIR EFFECT ON THE DYNAMICAL EVOLUTION}

The renormalization approach in statics is based on the idea of expressing the free energy of the initial system as that of a transformed, coarse-grained system, with different interactions, but with the same functional form of the free energy per particle. ${ }^{(1)}$ Such a relation between the free energies of the two systems is then expected to reduce, near criticality, to a realization of Widom's generalized homogeneity condition for the thermodynamic potential. In this way one is able to draw conclusions about both the location and the nature of the singularities displayed by the system. This information is obtained from the simple transformation properties of the free energy, and not from an explicit calculation of this function.

In Paper I we discussed a one-parameter dependent family of static transformations of the above-mentioned type. They were basically obtained by eliminating one of the $N$ spins in the summation (4) for the partition function. We computed the negative energy function $e^{\prime}(m)$ for a system of $N^{\prime}=N-1$ particles, up to first order in $N^{-1}$ as

$$
e^{\prime}=e+N^{-1}\left(e-m \dot{e}+\ln (2 \cosh \dot{e})+\frac{\alpha}{2}(m-\tanh \dot{e}) \sinh 2 \dot{e}-g\right)
$$

where $\alpha$ is a variable parameter and $g$ a normalization constant, independent of $m$, fixed, e.g., by the condition $e^{\prime}(0)=e(0)$. At the same time, the free energies per particle of the old and of the new system are related by the equation

$$
\beta F\left(e^{\prime}\right)=\left(1+N^{-1}\right) \beta F(e)+N^{-1} g
$$

It is essential for the discussion of the critical properties of the system that the new free energy has the same functional dependence on $e^{\prime}$ as the original free energy had on $e$.

The infinitesimal character of the transformation has many technical advantages, as discussed in I. A repeated application of the transformation reduces the number of spins to

$$
N^{\prime}=N e^{-s}
$$

and this induces a differential renormalization flow in the parameter $s$ for the negative energy function $e(m)$. Equation (16) can be rewritten as a partial differential equation for $e(m, s)$. The solution of this equation was treated in paper $I$.

In order to summarize a few properties of this differential equation which we will need in the present paper, let us remind that there is a critical 
fixed point solution given by

$$
\dot{e}^{*}(m)=\tanh ^{-1} m
$$

Furthermore, we can write

$$
e(m, s)=e^{*}(m)+\delta e=e^{*}(m)+\sum_{n} a_{n}(s) m^{n}
$$

For small deviations from $e^{*}$, we can linearize the differential equation in $\delta e$. The solution of this linearized renormalization equation is then given by

$$
a_{n}(s)=a_{n}(0) \exp ((1-n \alpha) s)
$$

By substituting (19) in (11), we find that $A\left(\dot{e}^{*}, m\right)$ is identically zero; this means that the relaxation time of the system is infinite at the fixed point, as it should be. In view of Eq. (12), it is evident that this basic property remains valid if we allow for transition probabilities different from those given by Eq. (6), as discussed in the previous section.

To get information about the critical properties of the dynamics of the model, we want to proceed with a strategy similar to the one followed in the statics. The basic idea is to use the static mapping induced by (16) in order to establish a transformation of the given dynamical evolution, described by $h$, into a new one, described by $h^{\prime}$, and governed by transition probabilities (6) connected to the renormalized energy function $e^{\prime}$. If we make sure that $h^{\prime}$ maintains the same functional form as $h$, it will be possible to extract information about the critical slowing down by only studying the established relation between $h$ and $h^{\prime}$, without solving explicitly for the time evolution of $h$.

Since the time evolution operator acting on $h$ depends on $\dot{e}$, it will be necessary for preserving the functional dependences that Eq. (10) for the evolution of $h$ transforms in a covariant way, i.e., we expect $h^{\prime}$ to satisfy an evolution equation

$$
\frac{\partial h^{\prime}}{\partial t^{\prime}}=A\left(\dot{e}^{\prime}, m\right) \dot{h}^{\prime}
$$

In Eq. $\left(10^{\prime}\right)$ we have put a prime in the time variable, in view of the fact that we expect, also on physical grounds, that the time variable must be rescaled in order to obtain an equation like $\left(10^{\prime}\right)$. In the following we will indeed show that Eq. $\left(10^{\prime}\right)$ can be established only at the cost of a rather complicated, $m$-dependent transformation of the time variable.

Consider a given $h$, solution of Eq. (10), specifying the deviation from equilibrium of the probability distribution (7) of our system with negative energy function $e(m)$. It is a straightforward procedure to calculate the effect of the infinitesimal transformation (16) on the function $e+h$. Keep- 
ing for $e^{\prime}$ the expression given by Eq. (16), we obtain, up to first order in $h$,

$$
h^{\prime}=h+N^{-1}(h+C \dot{h})
$$

where

$$
C=C(m, \dot{e}, \alpha)=\tanh \dot{e}-m-\frac{\alpha}{2} A(2 \dot{e}, m)
$$

It is clear that there corresponds to (22) a differential equation for $h$ with respect to the variable $s$ from (18). To obtain it, we have to write (22) in the form

$$
h^{\prime}=h+d s \frac{d L(s, \dot{e}, \alpha)}{d s} h
$$

where $L$ is a linear operator on the function $h$.

The function $h^{\prime}$, and in general $h\left(m, t, s_{0}\right)$, obtained by integrating Eq. (24) between 0 and $s_{0}$, specifies now the deviation from equilibrium of the renormalized probability distribution. Equations (22) and (24) constitute the basic relations for our dynamical mapping.

In order to obtain the time evolution equation for the renormalized function $h^{\prime}$, we combine (22) with (10) and we get, up to corrections of order $N^{-2,4}$

$$
\frac{\partial h^{\prime}}{\partial t}=A\left(\dot{e}^{\prime}, m\right) \dot{h}^{\prime}+N^{-1}(C \dot{A}-\dot{C} A) \dot{h}-\left(A\left(\dot{e}^{\prime}, m\right)-A(\dot{e}, m)\right) \dot{h}
$$

It is immediately clear that we do not yet obtain the desired covariance, as expressed in $\left(10^{\prime}\right)$. Our infinitesimal renormalization introduces in the dynamical equation some infinitesimal correction terms, which break the covariance. This is a general feature of the dynamical renormalization method, and it simply suggests to us that time itself has to be redefined in an appropriate nontrivial way, if we want to obtain some covariance property. Quite generally, we are thus looking for a new time variable $t_{R}(t, s, m)$ such that $\tilde{h}$, defined by

$$
\tilde{h}\left(m, t_{R}, s\right) \equiv h(m, t, s)
$$

satisfies for all $s$ the equation

$$
\frac{\partial \tilde{h}}{\partial t_{R}}=A(\dot{e}(m, s), m) \tilde{h}
$$

We may always impose $t_{R}(t, s=0, m)=t$. After the first infinitesimal

\footnotetext{
${ }^{4}$ In writing down Eq. (10), we did not include some additional correction terms of order $N^{-1}$, since they are not relevant to our discussion. (See, however, Ref. 4 for their importance in the study of metastable states.) Indeed, these terms should appear again in Eq. (25), with renormalization corrections of order $N^{-2}$.
} 
renormalization step $\left(d s=N^{-1}\right)$, we then have

$$
t_{R}=t+N^{-1} \beta \quad\left(\beta=\frac{\partial t_{R}}{\partial s}\right)
$$

Equation (25) can then be seen to reduce to the covariant form $\left(10^{\prime \prime}\right)$, if $\beta$ satisfies the equation

$$
\frac{\partial \beta}{\partial t_{R}}=A \frac{\partial \beta}{\partial m}+A^{-1}\left(C \dot{A}-\dot{C} A-\frac{\partial A}{\partial \dot{e}} \frac{d \dot{e}}{d s}\right) \equiv A \frac{\partial \beta}{\partial m}+\gamma
$$

As boundary condition for $\beta$, we may choose $\beta(m, t=0)=0$, which means that $t_{R}=0$ if $t=0$, and this for all $m$ and $s$. Looking for the full solution of (28) would of course be meaningless, since its homogeneous part is of the same form as (10), the equation which completely solves the dynamical problem. In the next section we will show how the dynamical critical properties, i.e., the properties of the solutions of (10) around the critical point, can be derived from the solution of (28) at the fixed point.

The full renormalized time $t_{R}$ can be obtained from $\beta$ by integration:

$$
t_{R}(m, t, s ;[e])=t+\int_{0}^{s} d s^{\prime} \beta\left(m, t_{R}\left(m, t, s^{\prime} ;[e]\right) ;[e]\right)
$$

where we pointed out the functional dependence of $t_{R}$ and $\beta$ on $e(m, s)$.

In this section we have thus achieved the result of mapping the linear dynamical evolution for $h$ as a function of $t$, which exists originally (i.e., for $s=0$ ), into an identical evolution at $s \neq 0$ for $\tilde{h}$ as a function of $t_{R}$. Indeed, one can easily check on the basis of the above definitions and results that the following property must hold for general $s$ :

$$
\begin{aligned}
& L(s,[e]) h\left(m, t,\left[h_{0}\right],[e(s=0)]\right) \\
& \quad=\tilde{h}\left(m, t_{R}(m, t, s,[e]),\left[L(s,[e]) h_{0}\right],[e(s)]\right)
\end{aligned}
$$

Here $h\left(m, t,\left[h_{0}\right],[e]\right)$ indicates the solution of the dynamical equation (10) for a given initial condition $h_{0}(m)$ at $t=0 . L(s,[e]) h$ represents the solution of the renormalization equations (22)-(24). From their definitions it should be clear that $L$ and $t_{R}$ depend functionally on $e\left(s^{\prime}\right)$ for all $s^{\prime}$ between 0 and $s$.

Equation (30) is the basic relation, which will allow us to investigate the properties of the dynamics near the fixed point.

\section{SCALING RELATION FOR THE RELAXATION TIME}

In this section, we first derive an expression for the renormalized time $t_{R}(m, t, s ;[e])$, asymptotically valid for large times $(t \rightarrow \infty)$ and for systems in the critical region. The fact that a system is in the critical region means 
that $e(m, s)$ will approximately become equal to $e^{*}(m)$ when $s$ becomes sufficiently large. ${ }^{5}$

The quantity $\gamma$ defined in (28) is given by

$$
\begin{aligned}
\gamma & =A^{-1}\left(C \dot{A}-\dot{C} A-\frac{\partial A}{\partial \dot{e}} \frac{d \dot{e}}{d s}\right) \\
& =-\ddot{e}\left(1-\tanh ^{2} \dot{e}\right)-\alpha \sinh ^{2} \dot{e}+2 \alpha \ddot{e}(\cosh 2 \dot{e}-m \sinh 2 \dot{e})
\end{aligned}
$$

At the fixed point solution (19), this reduces to

$$
\gamma^{*}=-1+2 \alpha+\alpha m^{2} /\left(1-m^{2}\right)
$$

Let us, for the time being, take the attitude that we are close enough to criticality to forget about the difference between $\gamma$ and $\gamma^{*}$. Furthermore, we will neglect the $m$ dependence of $\gamma$. The validity of this assumption may be intuitively argued from the fact that if we let $t$ become sufficiently large, the system will relax almost completely to its equilibrium state, in which the average magnetization is zero, or nearly zero, when close to criticality. Therefore, we expect that the dominant features of the critical behavior must be controlled by what happens around the point $m=0$. We will show in the present section how this simplistic approach leads to the correct critical dynamical behavior. A more complete justification of these assumptions is reported in the Appendix, where a detailed investigation is made of some basic properties of the solutions for $\beta$ and $t_{R}$ near criticality.

Since at criticality we have that $A=0$, in our approximations (28) leads immediately to

$$
\beta \simeq-(1-2 \alpha) t_{R}
$$

and from (29)

$$
t_{R} \simeq t \exp [-(1-2 \alpha) s]
$$

It may be expected from the nature of our simplifications that this solution will be asymptotically correct in the critical region and for large enough times.

Let us now look at the consequences of the time renormalization (34), by using the basic relation (30). This equation expresses the fact that time evolution and renormalization ( $s$ evolution) are commutative operations, when we use the proper renormalized time. It is natural to assume as general form for the time-dependent solution of Eq. (10) a superposition of exponentially decaying modes:

$$
h\left(m, t,\left[h_{0}\right],[e]\right)=\sum_{k} \lambda_{k}\left(\left[h_{0}\right]\right) H_{k}(m) \exp \left\{-t / t_{k}([e])\right\}
$$

\footnotetext{
${ }^{5}$ We have in mind the particular choice $1 / 3<\alpha<1 / 2$, which in view of (21) yields only the magnetic field and the temperature as relevant variables in the traditional sense.
} 
$H_{k}(m)$ are the different modes that relax to zero at equilibrium. Since both the initial condition $h_{0}(m)$ and the energy function $e(m)$ are influenced by the renormalization and are thus $s$ dependent, we may expect that in the right-hand side of Eq. (30) $\lambda_{k}, H_{k}(m), t$, and the relaxation times $t_{k}$ will all be $s$ dependent. Our main interest lies, however, in the critical behavior of $t_{0}$, the relaxation time of the slowest mode. On the basis of Eq. (35), relation (30) may be rewritten as

$$
\begin{aligned}
\sum_{k} \lambda_{k}\left(\left[h_{0}\right]\right) \exp \left\{-t / t_{k}([e(s=0)])\right\} L(s,[e]) H_{k}(m, s=0) \\
=\sum_{k} \lambda_{k}\left(\left[L(s,[e]) h_{0}\right]\right) H_{k}(m, s) \exp \left\{-t_{R}(t, s,[e]) / t_{k}([e(s)])\right\}
\end{aligned}
$$

If both $t$ and $t_{R}$ become very large, only the slowest mode is of importance, and the identity of the corresponding terms leads us immediately to

$$
\frac{t}{t_{0}([e(s=0)])}=\frac{t_{R}(t, s,[e])}{t_{0}([e(s)])}
$$

or, with (34),

$$
t_{0}([e(s)])=e^{-(1-2 \alpha) s} t_{0}([e(s=0)])
$$

If we characterize the function $e(m, s)$ by the coefficients $a_{n}$ defined in (20), we obtain the scaling relation

$$
t_{0}\left(\left\{a_{n}\right\}\right)=e^{(1-2 \alpha) s} t_{0}\left(\left\{a_{n} e^{(1-n \alpha) s}\right\}\right)
$$

or, by defining

$$
\begin{aligned}
& \lambda=e^{(1-2 \alpha) s} \\
& p=e^{-\alpha s}
\end{aligned}
$$

this is equivalent to

$$
t_{0}\left(\left\{a_{n}\right\}\right)=\lambda t_{0}\left(\left\{\lambda p^{n-2} a_{n}\right\}\right)
$$

This equation for the relaxation time may be analyzed in a similar way as the scaling relation for the free energy in Paper I. As relevant application, let us again look at the case where only three parameters are important: $a_{1}=h, a_{2}=\tau \sim T-T_{c}$, and $a_{4}$. We then have

$$
t_{0}\left(h, \tau, a_{4}\right)=\lambda t_{0}\left((\lambda / p) h, \lambda \tau, \lambda p^{2} a_{4}\right)
$$

The $p$ independence of the left-hand side implies that $t_{0}$ is a function of $\tau$ and $\left(h^{2} a_{4}\right)$ only, and thus

$$
t_{0}\left(h, \tau, a_{4}\right)=f\left(\tau, h^{2} a_{4}\right)=\lambda f\left(\lambda \tau, \lambda^{3} h^{2} a_{4}\right)
$$

This means that the relaxation time $t_{0}$ should be homogeneous of order 
$(-1)$ in $\tau$ and $\left(h^{2} a_{4}\right)^{1 / 3}$, or

$$
\begin{array}{cc}
t_{0} \sim\left|T-T_{c}\right|^{-1} & \text { for } h=0 \\
\sim h^{-2 / 3} & \text { for } T=T_{c}
\end{array}
$$

Both results may be understood from standard mean field arguments.

We end this section by noticing that, if we would consider transition probabilities different from (6), and leading to time evolution operator coefficients given by Eq. (12), we would get in the expression for $\gamma$ a correction term equal to $C \dot{a} / a$. Since at the fixed point $C$ is equal to zero for $m=0$, the above conclusions are expected to hold also in this more general case. The same kind of argument can still be seen to apply if we allow the function $a(m)$ to depend on $m$ also through the argument $\dot{e}(m)$, i.e., if we make $a(m)$ temperature dependent. In this case, of course, also the term $\partial A / \partial \dot{e} \cdot d \dot{e} / d s$ in $\gamma$ is modified, but one can again check that the difference becomes zero at $m=0$ for $\dot{e}=\dot{e}^{*}$. This result provides an explicit example of a very simple mechanism of stability of the dynamical critical behavior with respect to modifications of the transition rate in the master equation.

\section{CONCLUDING REMARKS}

Even if strongly limited by the mean field character of the model, the approach presented here has, in our opinion, some remarkable features. A few of these features give clear indications of problems and properties which arise in more general and difficult contexts.

As a first striking property, we would like to mention the $m$ dependence of the renormalized time function $t_{R}$. This $m$ dependence, which turns out to be essential in establishing covariance in the equation of motion, is rather notable because it implies that time itself must become a random variable, on the same footing as $m$ itself. In the case of short-range forces, one should thus by analogy expect that the time transforms under renormalization into a function of the spin configuration of the system. This is of course somewhat discouraging. To handle, even approximately, such a configurational dependence of the time will be a very hard task, certainly if one wants to embody this dependence in a reasonably simple global scheme of dynamical evolution, like that provided by the master equation.

A rather interesting mechanism revealed by our analysis is the one discussed in detail in the Appendix. In our opinion it is of crucial importance, because it shows how some very general features of the dynamics of the system are at the basis of the possibility of recovering the appropriate 
dynamical exponents of the system by renormalization methods. Indeed, in the Appendix we show that the whole $m$-dependent part of $\gamma$ is irrelevant in determining the asymptotic large time behavior of $\beta$, and thus of $t_{R}$. This is due to the circumstance that the average magnetization relaxes to zero at criticality, irrespective of the detailed way in which this relaxation occurs.

Another aspect that we were able to put directly in evidence is the detailed mechanism which leads in the dynamical critical behavior to the universality with respect to the choice of the transition rate within a rather wide class. The only effect of the modification of the transition rate is in the transient effects, occurring out of the asymptotic time regime, where scaling is expected (see Appendix).

In closing our discussion, we want to call attention to the fact that a rather striking characteristic of the approach presented here is its relatively high degree of complexity, which one would not a priori expect when dealing with such simple systems as mean field models. This is once more a general, very eloquent indication of the serious difficulties involved in the realization of an equivalent renormalization approach to the dynamics of more realistic models.

\section{APPENDIX}

The equations (28)-(29) for the renormalized time have been solved straightforwardly in Section 4 on the basis of two qualitatively justified assumptions: (a) the $m$-dependent terms in $\gamma$ can be ignored, at least for sufficiently large times; and (b) for the calculation, the system is assumed to relax to its fixed-point equilibrium state, neglecting deviations from $e^{*}$. In this appendix, we show that these assumptions are indeed legitimate.

\section{A.1. The $m$-Dependent Terms}

We show here that, for $e$ close to $e^{*}$, the contribution to $\beta$ of the $m$-dependent terms in $\gamma$ remains finite when $t_{R}$ goes to infinity.

We start by noticing a basic property of the function $A(\dot{e}, m)$ [given by Eq. (11) or (12)] which enters in the time evolution equation (10) of the system. If we denote by $S(m)$ the entropy function, already defined in Paper I,

$$
S(m)=-\int d m \tanh ^{-1} m
$$

we verify that, in general, at each $\bar{m}$ for which

$$
\dot{S}(\bar{m})+\dot{e}(\bar{m})=0
$$


we also have

$$
A(\dot{e}, \bar{m})=0
$$

The $m$ values which satisfy (A.2) correspond to extremal points for the equilibrium probability distribution of the $m$ variable. This means that, irrespective of its detailed behavior, $A(\dot{e}, m)$ always vanishes at the $\bar{m}$ value which corresponds to the absolute maximum of that probability distribution function. In the thermodynamic limit, this value coincides with the average magnetization, as follows immediately from a saddle-point argument.

Let us now consider, as an example, a situation for $T>T_{c}$, with eventually a magnetic field present. The typical shape of $-A$ as a function of $m$ is sketched in Fig. 1. We expect only one zero at $\bar{m}$, coinciding with the origin in the case of zero external field. Secondary maxima are indeed excluded by the absence of metastable states for $T>T_{c}$.

The solution of Eq. (28) can be written in terms of the functions $\tilde{m}\left(\tilde{m}_{0}, t\right)$ and $\tilde{m}_{0}(\tilde{m}, t)$, which are, respectively, the solution of the characteristic equation (14) and its inverse. We have, namely,

$$
\beta(m, t)=\int_{0}^{t} d t^{\prime} \gamma\left(\tilde{m}\left(\tilde{m}_{0}(m, t), t^{\prime}\right)\right)
$$

We have suppressed in (A.4) the functional dependences on $e(m)$, in order to simplify the notation.

Due to the shape of $A$, it is easily recognized that $\tilde{m}_{0}(m, t)$ will tend to $\bar{m}$ when $t$ goes to infinity, whatever is the detailed behavior of $\tilde{m}_{0}$. This reflects the fact that the mean magnetization approaches $\bar{m}$ for $t$ going to infinity. The same property holds for $\tilde{m}\left(\tilde{m}_{0}(m, t), t^{\prime}\right)$ for every finite value of $t^{\prime}$. This property now suggests to us splitting up $\beta$ into two pieces as follows:

$$
\beta(m, t)=\int_{0}^{t} d t^{\prime} \gamma(\bar{m})+\int_{0}^{t} d t^{\prime}\left(\gamma\left(\tilde{m}\left(\tilde{m}_{0}(m, t), t^{\prime}\right)\right)-\gamma(\bar{m})\right)
$$

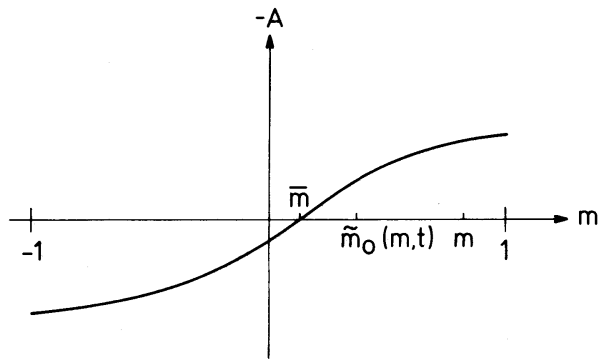

Fig. 1. Typical behavior of the function $-A(\dot{e}, m)$ for a situation above criticality and in the presence of a magnetic field. If the magnetic field becomes zero, $\bar{m}$ coincides with the origin. 
By a simple change of variable one can check that the second integral must be bounded, for every time $t$. Indeed, we can put it in the form

$$
-\int_{\tilde{m}_{0}(m, t)}^{m} d x A^{-1}(x)(\gamma(x)-\gamma(\bar{m}))
$$

Since $\tilde{m}_{0}(m, t)$ approaches $\bar{m}$ for $t$ going to infinity, this integral remains bounded (for reasonable behaviors of $\gamma$ and $A$ around $\bar{m}$ ), at least for $\dot{e} \neq \dot{e}^{*}$. At the fixed point itself, the integral diverges, since at $e^{*}$ we have that $A \equiv 0$. This means that for long times and for $T \gtrsim T_{c}$ the asymptotic value of $\beta$ is always given by

$$
\beta(m, t) \underset{t \rightarrow \infty}{\sim} \gamma(\bar{m}) t
$$

This result tells us that indeed the qualitative discussion of Section 4 on the asymptotic behavior of the renormalized time function was substantially correct, at least above criticality. We must indeed take into account the fact that $\bar{m}$ must approach zero when the system becomes critical (i.e., at zero magnetic field).

The discussion for $T<T_{c}$ is complicated by the presence of metastable states, which would amount to the presence of additional zeros for the function $A(m)$. The basic arguments are the same as above, and we omit the full discussion.

The transient time effects implied by the presence of terms of the form (A.6) in the behavior of $\beta$ will obviously depend on the type of transition rate we actually choose in the master equation, according to our considerations of Section 2.

\section{A.2. The Deviations from the Fixed Point}

We have just shown how we need a small deviation from criticality in order to prove the irrelevance of the $m$ dependence of the renormalized time. Nevertheless, also the explicit dependence on $\delta \dot{e}$ of $\gamma$ may be neglected, in the sense that it can only lead to corrections to scaling for the relaxation time $t_{0}$.

The irrelevant scaling fields may of course be neglected by letting $s$ tend to infinity. Let us put $h=0$ and restrict our argument to the dependence on the temperature parameter $a_{2}=\tau$. From (39) one can immediately derive result (44), i.e., the inverse proportionality of the relaxation time to the deviation from the critical temperature. We will show how this result is changed if one includes the explicit $a_{2}$ dependence of $\gamma$.

From (31) one may show that

$$
\gamma(m=0)=-(1-2 \alpha)\left(1+2 a_{2}\right)
$$

which is correct up to terms of order $a_{2}^{2}$. Since $a_{2}$ scales according to (21), 
this leads us to

$$
\frac{\partial t_{R}}{\partial s}=-t_{R}(1-2 \alpha)\left[1+2 a_{2} e^{(1-2 \alpha) s}\right]
$$

This equation has the solution

$$
t_{R}\left(m=0, t, s,\left[e=e^{*}+a_{2} m^{2}\right]\right)=t \exp \left\{-\left[(1-2 \alpha) s+2 a_{2} e^{(1-2 \alpha) s}\right]\right\}
$$

With the same argument that leads from (39) to (44), this gives us for the relaxation time the scaling relation

$$
t_{0} \sim\left|T-T_{c}\right|^{-1} \exp \left(-2\left|T-T_{c}\right|\right)
$$

The exponential is of course a negligible correction term. A similar argument may be given in the presence of a magnetic field.

\section{REFERENCES}

1. Th. Niemeijer and J. M. J. van Leeuwen, in Phase Transitions and Critical Phenomena, C. Domb and M. S. Green, eds., Vol. 6, p. 425 (Academic Press, London, 1976).

2. G. F. Mazenko, in Dynamical Critical Phenomena and Related Topics, Proc. of the International Conference of Geneva, 1979, C. P. Enz, ed., p. 97 (Springer, Berlin, 1979). This report also contains references on recent related work.

3. R. Dekeyser and A. Stella, J. Stat. Phys. 23:587 (1980).

4. R. B. Griffiths, C.-Y. Weng, and J. S. Langer, Phys. Rev. 149:301 (1966).

5. Ph. A. Martin, J. Stat. Phys. 16:149 (1977). 